\title{
Nonmonotonic Aging and Memory Retention in Disordered Mechanical Systems
}

\author{
Yoav Lahini, Omer Gottesman, Ariel Amir, and Shmuel M. Rubinstein \\ Harvard John A. Paulson School of Engineering and Applied Sciences, Harvard University, \\ Cambridge, Massachusetts 02138, USA
}

(Received 16 August 2016; revised manuscript received 18 November 2016; published 21 February 2017)

\begin{abstract}
We observe nonmonotonic aging and memory effects, two hallmarks of glassy dynamics, in two disordered mechanical systems: crumpled thin sheets and elastic foams. Under fixed compression, both systems exhibit monotonic nonexponential relaxation. However, when after a certain waiting time the compression is partially reduced, both systems exhibit a nonmonotonic response: the normal force first increases over many minutes or even hours until reaching a peak value, and only then is relaxation resumed. The peak time scales linearly with the waiting time, indicating that these systems retain long-lasting memory of previous conditions. Our results and the measured scaling relations are in good agreement with a theoretical model recently used to describe observations of monotonic aging in several glassy systems, suggesting that the nonmonotonic behavior may be generic and that athermal systems can show genuine glassy behavior.
\end{abstract}

DOI: 10.1103/PhysRevLett.118.085501

Many disordered systems exhibit phenomenologically similar slow relaxation dynamics that may span many time scales-from fractions of a second to days and even years. Examples range from time-dependent resistivity in disordered conductors [1-5], flux creep in superconductors [6,7], dynamics of spin glasses [8-11], structural relaxation of colloidal glasses $[12,13]$, time dependence of the static coefficient of friction [14-16], thermal expansion of polymers [17,18], compaction in agitated granular systems [19], and crumpling of thin sheets under load [20,21]. The ubiquity of slow relaxation phenomena suggests the existence of common underlying physical principles [9,22-27]. However, as slow relaxation is usually a smooth, featureless process, it is hard to discern between the different descriptions using experiments. One way of probing deeper into the time-dependent properties of glassy systems is using a phenomenon known as aging, where the manner in which the system relaxes towards equilibrium depends on its history.

In this Letter, we report nonmonotonic aging dynamics that give rise to a maximum in the relaxation curve. This extremum provides an unambiguous signature of aging and memory, as well as a clear, measurable time scale. We experimentally study two distinct disordered mechanical systems: crumpled thin sheets and elastic foams, shown in Fig. 1. When compressed, both systems exhibit monotonic, slow stress relaxation [Figs. 1(b) and 1(e)]. When the compression is decreased after a certain waiting time, the stress evolution remarkably becomes nonmonotonic: under constant compression, the measured normal force first increases slowly over seconds to hours, reaches a welldefined peak, and then reverses to a renewed slow relaxation [Figs. 1(c) and 1(f)]. In both systems, the stress peak time is linear in the waiting time, indicating that the different systems carry a similar, long-lasting memory of previous mechanical states. These observations are inconsistent with the single-parameter model used to explain logarithmic relaxation in crumpled sheets [21], yet are in agreement with a different phenomenological framework, successfully used recently to define a new universality class related to the generic behavior of aging in several glassy systems [27].

Slow relaxation and aging experiments are performed in a custom uniaxial compression tester. Samples are compressed between two parallel plates, separated by a gap $H$, which is set by a motorized stage. The compressive normal force $F_{N}$ is monitored using an $S$-beam load cell (Futek LSB200) acquired at $24 \mathrm{kHz}$. We measure the stress relaxation behavior of thin Mylar sheets, $33 \mathrm{~cm} \times 33 \mathrm{~cm} \times 15 \mu \mathrm{m}$, crumpled into a ball, as shown in Fig. 1(a). Samples are placed between the plates of the apparatus, separated by an initial gap of $H_{1}$. The gap is then reduced to $H_{2}<H_{1}$ and is held constant for the rest of the experiment. Under these conditions, the crumpled sheets exhibit logarithmic stress relaxation, as shown for a typical example in Fig. 1(b). Such behavior was observed by Matan et al. [21] and later by others [28]. Similar slow relaxations spanning several decades in time are exhibited by samples of elastic foam: dense open-cell porous materials made of elastic PVC, $18 \mathrm{~mm}$ in height and $10 \mathrm{~mm}$ in diameter, shown in Fig. 1(d).

We perform a comprehensive set of stress relaxation tests on both materials, keeping $H_{1}$ constant and measuring relaxation curves for different compressions $\delta=H_{1}-H_{2}$. For crumpled Mylar, we quantify the relaxation by fitting the curve to $F_{N}(t)=a+b \log (t)$. Here, $a$ is related to the normal force measured one second after the compression and $b$ is the logarithmic relaxation rate. Typically, for larger compression steps, both $a$ and $b$ are larger. However, as 

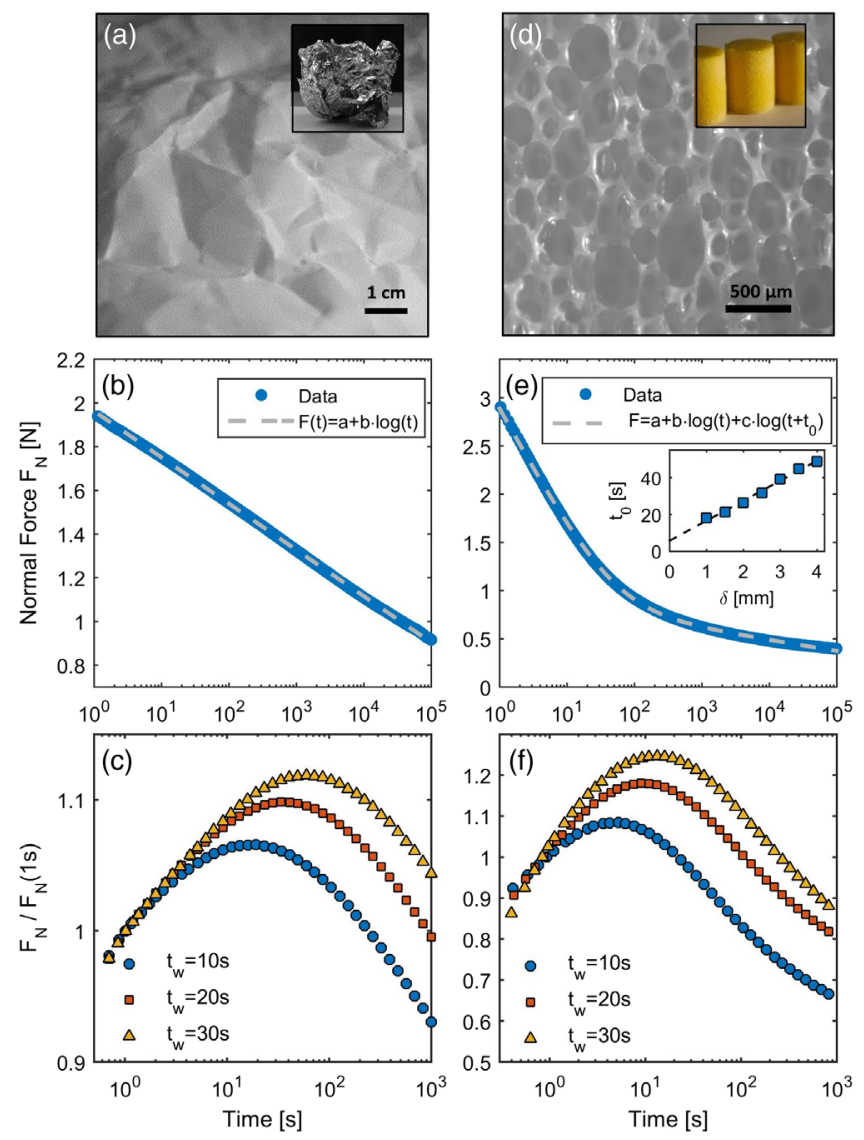

FIG. 1. Two disordered mechanical systems. (a) The network of creases decorating a crumpled sheet of Mylar. Inset: a crumpled Mylar ball. (b) Stress relaxation of the crumpled ball with $H_{1}=45 \mathrm{~mm}, \delta=H_{1}-H_{2}=5 \mathrm{~mm}$. The dashed line is a fit to a logarithmic decay. (c) Nonmonotonic relaxation of crumpled Mylar, initially compressed by $\delta=5 \mathrm{~mm}$ for $t_{w}$ and then released by $\Delta=2 \mathrm{~mm}$. (d) Typical microscopic image of the cross section of a PVC foam. Inset: elastic foam samples. (e) Stress relaxation of elastic foam with $H_{1}=18 \mathrm{~mm}$ and $\delta=4 \mathrm{~mm}$. The dashed line is a fit to $F=a+b \log (t)+c \log \left(t+t_{0}\right)$. Inset: $t_{0}$ vs $\delta$. (f) Nonmonotonic relaxation for elastic foam with $\delta=3 \mathrm{~mm}$ and $\Delta=1.5 \mathrm{~mm}$.

reported in previous work on relaxation in crumpled sheets [21], we find that the relaxation curves fluctuate strongly between runs and no systematic relation appears between $\delta$, $a$, and $b$. This irreproducibility hampers any attempt to quantify the slow relaxation and the more subtle aging behavior reported below. To this end, we identify an experimental procedure in which the randomly crumpled sheets are "trained" before the experiments and as a result yield reproducible behavior. First, new sheets of Mylar are repeatedly crumpled, opened, and flattened. To achieve the same maximum compaction during training, the sheets were always crumpled into a cylinder, $65 \mathrm{~mm}$ in diameter and $35 \mathrm{~mm}$ in height. After at least 30 iterations, additional crumpling of the sheet creates very few new creases [29]. Second, before each experiment we perform a quick compression and release of the crumpled ball. Finally,
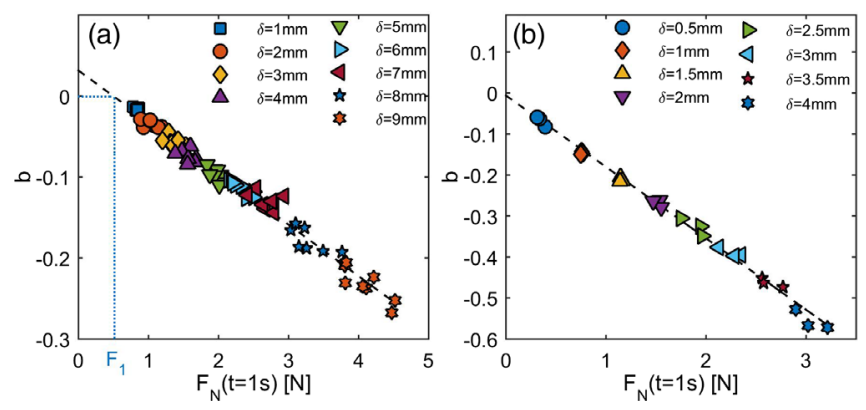

FIG. 2. Reproducible stress relaxation. (a) Crumpled Mylar: The relaxation rate $b$ vs the normal force at $t=1 \mathrm{~s}$, $F(t=1 \mathrm{~s})=a$. The different symbols represent experiments performed with different values of $\delta$. The normal force for which $b=0$ is marked as $F 1$ (dotted blue line). (b) Same as (a) for elastic foams, where here $F_{N}(t=1 \mathrm{~s})=a+c \log \left(t_{0}+1\right)$ and we fit each relaxation curve to $F_{N}(t)=a+b \log (t)$ $+c \log \left(t+t_{0}\right)$.

$H_{1}=45 \mathrm{~mm}$ is used for the crumpled sheets, as we found it to be the maximal gap beyond which the crumpled ball could slip out of the apparatus. The normal force measured after training, which we denote as $F_{1}$, is approximately $0.5 \mathrm{~N}$ in all experiments. The corresponding relaxation rate, however, is negligible [see Fig. 2(a)]. The elastic foams do not require any support; thus, here $H_{1}$ is the height of the sample. The value for maximum compression $\mathrm{H}_{2}=5 \mathrm{~mm}$ for the crumpled sheet and $H_{2}=3 \mathrm{~mm}$ for the elastic foams was chosen such that the experiments remain in the linear strain-stress regime. For higher compressions, we observed a transition in to a power-law dependence [21,30-32]. Under these conditions, we observe reproducible logarithmic relaxation curves, as shown in Fig. 2(a). In particular, we find a linear relation between $b$ and $F(1 \mathrm{~s})$ that is offset by $F_{1}$. The elastic foams require no training; measurements are reproducible as long as the sample is allowed to relax back to its original state between tests. Here, the relaxation curves for all compressions $\delta$ can be fitted to a double-logarithmic function of the form $F_{N}=a+b \log (t)+c \log \left(t+t_{0}\right) . \quad b$ and $c$ are proportional to $\delta$, while the ratio between them remains approximately constant over all the relaxation curves. The inherent time scale $t_{0}$ shows a linear dependence on $\delta$, as shown in the inset of Fig. 1(e).

The reproducible relation between the compression $\delta$ and the relaxation rate enables a systematic investigation of the more subtle aging and memory effects which are observed after a sequence of compressions. Usually, the notion of aging implicitly assumes a slow monotonic process; however, in both systems we find that a two-step compression protocol results in nonmonotonic aging dynamics and memory effects. Here, a sample is placed between the two plates of the apparatus, separated by a gap $H_{1}$; the gap is then decreased to $H_{2}<H_{1}$ and held constant for a specific waiting time $t_{w}$. During this first step, the normal force monotonically decreases. At $t=t_{w}$, the gap between 


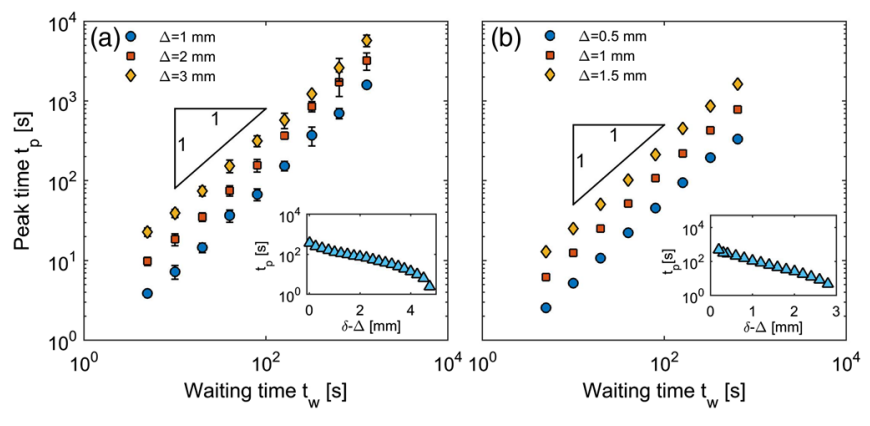

FIG. 3. Memory effect. Linear scaling between the peak time and the waiting time for different values of $\Delta$, shown for (a) crumpled thin sheets and (b) elastic foams. Insets: peak time vs $H_{1}-H_{3}$ for $t_{w}=20 \mathrm{~s}$.

the plates is increased to $H_{3}$ such that $H_{2}<H_{3}<H_{1}$ and held constant for the rest of the experiment. The subsequent dynamics separate into three distinct stages. First, during the gap increase from $\mathrm{H}_{2}$ to $\mathrm{H}_{3}, F_{N}$ shifts abruptly to a lower value due to an elastic response of the samples. Subsequently, in contrast to the naive expectation that $F_{N}$ should now decrease at a logarithmic rate that corresponds to the new compression, the normal force exhibits a slow, nonmonotonic behavior. Under constant external conditions, $F_{N}$ first slowly increases over many minutes and even hours. $F_{N}$ reaches a well-defined force peak at a time $t_{p}$, after which, for $t>t_{p}, F_{N}$ crosses back to a slow decay. Very similar nonmonotonic dynamics are measured for both systems, as shown for crumpled Mylar sheets in Fig. 1(c) and elastic foams in Fig. 1(f). We note that these systems also show nonmonotonic volume relaxation when subjected to a two-step loading protocol (not shown).

At any two time points in the nonmonotonic dynamics in which $F_{N}$ has the same value before and after the peak, the sample's compression and all other macroscopic observables are identical; however, the system's evolution at these two points is qualitatively different. Thus, the nonmonotonic behavior clearly indicates that the state of the system cannot be described by the macroscopic observables alone, and additional degrees of freedom storing a memory of the system's history must exist.

To characterize this nonmonotonic behavior, we performed a systematic study of the relation between the peak time $t_{p}$, the waiting time $t_{w}$, and the change in compression at the last stage $\Delta=\mathrm{H}_{3}-\mathrm{H}_{2}$. Here, the reproducibility of the experiments is crucial. For fixed values of $\Delta$, the relation between the waiting time $t_{w}$ and the peak time $t_{p}$ is approximately linear over several decades. Increasing $\Delta$ results in a steeper linear dependence. These results are depicted in Fig. 3(a) for the crumpled sheets and Fig. 3(b) for elastic foams. Additional measurements in which $t_{w}$ was kept constant while $\Delta$ was varied over a wide range indicate that the peak time increases as $H_{3}$ approaches $H_{1}$, as shown in the insets of Figs. 3(a) and 3(b).
The scaling between $t_{w}$ and $t_{p}$ is a hallmark of a memory effect-the time in which the system reached its peak normal force is correlated with changes in external conditions made up to several hours earlier. These observations rule out single degree of freedom descriptions previously suggested to model slow relaxations in several disordered systems [7,33], including crumpled thin sheets [21]. Singleparameter theories relate the relaxation rate of some macroscopic observable to its instantaneous value and thus cannot account for nonmonotonic behavior or for historydependent evolution-i.e., memory. An alternative phenomenological framework was recently used successfully to describe aging in several glassy systems, introducing a new universality class related to the generic behavior of logarithmic aging [27]. We show that this framework can be generalized to apply also to the experiments discussed here, capturing both the nonmonotonic relaxation as well as the observed linear scaling between $t_{p}$ and $t_{w}$. We assume a system which is controlled by a single parameter $E$ and which evolves via an ensemble of independent exponential relaxation modes, each characterized by a rate $\lambda$ with a broad distribution of rates $P(\lambda)$. A key assumption is that for every $E$ there exists an equilibrium state $V^{\mathrm{eq}}$ and that all relaxation modes have the same amplitude and thus contribute to it equally. If a system is initially at the equilibrium state $V_{1}^{\mathrm{eq}}$ when $E$ is switched to a different value, its relaxation towards a new equilibrium $V_{2}^{\mathrm{eq}}$ can be written as

$$
V(t)=V_{2}^{\mathrm{eq}}+\left(V_{1}^{\mathrm{eq}}-V_{2}^{\mathrm{eq}}\right) \int_{\lambda_{\min }}^{\lambda_{\max }} P(\lambda) e^{-\lambda t} d \lambda,
$$

where $\lambda_{\min }$ and $\lambda_{\max }$ are physical cutoff rates. Specifically, for $P(\lambda) \propto 1 / \lambda$ and $1 / \lambda_{\max } \ll t \ll 1 / \lambda_{\min }$, we recover the logarithmic relaxation observed for the crumpled balls: $V(t)=V_{2}^{\mathrm{eq}}-\left(V_{1}^{\mathrm{eq}}-V_{2}^{\mathrm{eq}}\right)\left[\gamma_{E}+\log \left(\lambda_{\min } t\right)\right] \equiv a+b \log (t)$, where $\gamma_{E}$ is the Euler-Mascheroni constant. This particular distribution was shown to arise in certain disordered systems via several potential mechanisms [27], including thermal activation (also leading to $1 / f$ noise $[34,35]$ ) and multiplicative processes. Interestingly, the same distribution also arises in the context of "sloppy models" [36] and random matrices [37,38].

This formalism can predict the observed nonmonotonic relaxations, without additional assumptions. Here, starting at equilibrium $V_{1}^{\mathrm{eq}}$, the system evolves towards $V_{2}^{\mathrm{eq}}$ only for a finite time $t_{w}$-as shown schematically in Fig. 4(a). At this point, the equilibrium state shifts to $V_{3}^{\mathrm{eq}}$. If $V_{1}^{\mathrm{eq}}>V_{3}^{\mathrm{eq}}>V_{2}^{\mathrm{eq}}$, then at $t=t_{w}$, different modes can be found at different sides of the equilibrium, as shown Fig. 4(b). The slow modes, with decay rate $\lambda \ll 1 / t_{w}$, are still in the vicinity of $V_{1}^{\text {eq }}$, i.e., above $V_{3}^{\text {eq }}$, while the fast modes with $\lambda \gg 1 / t_{w}$ have reached the new equilibrium $V_{2}^{\mathrm{eq}}$ and are below $V_{3}^{\mathrm{eq}}$. Thus, immediately after $t_{w}$ the dynamics of the fast and slow modes are in opposite directions. At this stage, the overall response can be 


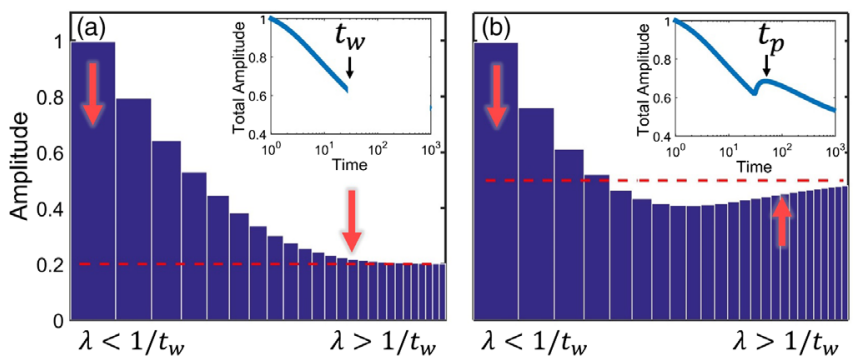

FIG. 4. Phenomenological model. Simulation of Eq. (3) for $V_{1}^{\mathrm{eq}}=1, V_{2}^{\mathrm{eq}}=0.2, V_{3}^{\mathrm{eq}}=0.5$, and $t_{w}=30$. The instantaneous amplitude of the relaxation modes are shown for (a) $t=t_{w}$ and (b) $t=t_{p}$. The slow modes with $\lambda>1 / t_{w}$ and the fast modes with $\lambda<1 / t_{w}$ are depicted on the left and right, respectively. The width of the bars represents the abundance of the different relaxation times according to $P(\lambda) \propto 1 / \lambda$. Insets: total amplitude as a function of time calculated by summing over all the individual modes.

dominated by the fast modes, and as a result $V(t)$ increases over time [Fig. 4(b)]. After the fast modes reach the new equilibrium, the overall response is dominated by the slow modes, leading to resumed relaxation.

Equation (1) can be generalized for multiple steps by accounting for the out-of-equilibrium state of each mode at time $t_{w}$. At this time, the state of each relaxation mode is given by $V_{3, \lambda}(t)=V_{3, \lambda}^{\mathrm{eq}}+\left[V_{2, \lambda}\left(t_{w}\right)-V_{3, \lambda}^{\mathrm{eq}}\right] e^{-\lambda t}$ with $V_{2, \lambda}\left(t_{w}\right)=V_{2, \lambda}^{\mathrm{eq}}+\left(V_{1, \lambda}^{\mathrm{eq}}-V_{2, \lambda}^{\mathrm{eq}}\right) e^{-\lambda t_{w}}$. Thus, for $t>t_{w}$, the system's evolution is given by

$$
\begin{aligned}
V_{3}(t)= & V_{3}^{\mathrm{eq}}+\left(V_{2}^{\mathrm{eq}}-V_{3}^{\mathrm{eq}}\right) \int_{\lambda_{\min }}^{\lambda_{\max }} P(\lambda) e^{-\lambda t} d \lambda \\
& +\left(V_{1}^{\mathrm{eq}}-V_{2}^{\mathrm{eq}}\right) \int_{\lambda_{\min }}^{\lambda_{\max }} P(\lambda) e^{-\lambda\left(t+t_{w}\right)} d \lambda .
\end{aligned}
$$

As before, this expression can be approximated by:

$$
\begin{aligned}
V_{3}(t)= & V_{3}^{\mathrm{eq}}-\left(V_{2}^{\mathrm{eq}}-V_{3}^{\mathrm{eq}}\right)\left[\gamma_{E}+\log \left(\lambda_{\min } t\right)\right] \\
& -\left(V_{1}^{\mathrm{eq}}-V_{2}^{\mathrm{eq}}\right)\left\{\gamma_{E}+\log \left[\lambda_{\min }\left(t+t_{w}\right)\right]\right\} .
\end{aligned}
$$

Turning back to the experiments, the equilibria values $V^{\text {eq }}$ represent the normal forces as would be measured at infinitely long time, where the equilibrium force is larger for lower $V^{\text {eq }}$. However, this regime is not attainable experimentally as the normal forces we measure do not show any signs of reaching equilibrium. Nevertheless, according to the model, $b$ is proportional to changes in the $V^{\text {eq }}$, so that $V_{2}^{\mathrm{eq}}-V_{3}^{\mathrm{eq}} \propto b_{2}-b_{3}$, etc. As shown in Fig. 2(a), for the crumpled Mylar sheets $b$ is proportional to $F(t=1 \mathrm{~s})-F_{1}$. Thus, we can replace $b_{i}$ with $F_{i}-F_{1}=F\left(H_{i}\right),(i \in 2,3)$, where $F_{i}$ is the normal force as measured one second after a compression from $H_{1}$ to $H_{i}$, and $F_{1}$ is the normal force measured at $H_{1}$ just before the compression. Using this substitution and by differentiating Eq. (3) to find the curve maximum, we find
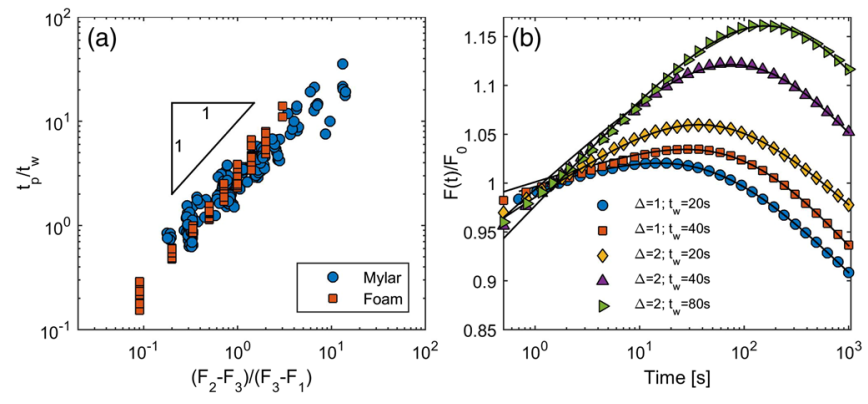

FIG. 5. Universal relaxation dynamics. (a) $t_{p} / t_{w}$ vs $\left(F_{2}-F_{3}\right) /\left(F_{3}-F_{1}\right)$ for all the experiments performed on crumpled sheets (blue circles) and elastic foams (red squares). (b) Single-parameter fits for the nonmonotonic relaxation of Mylar sheets to $A-B\left[\left(F_{3}-F_{2}\right) \log (t)+\left(F_{2}-F_{1}\right) \log \left(t+C t_{w}\right)\right]$ with $B=A \times 0.025$ and $C=2.55$.

$$
t_{p} / t_{w}=\left(F_{2}-F_{3}\right) /\left(F_{3}-F_{1}\right) .
$$

Using this scaling relation, the data from all experiments performed on crumpled Mylar approximately collapses to a single linear curve as shown in Fig. 5(a). The analysis reveals an additional constant, denoted as $C$, such that the collapsed curve is of the form $t_{p} / t_{w}=C\left(F_{2}-F_{3}\right) /\left(F_{3}-F_{1}\right)$ with $C=2.6 \pm 0.2$. Accordingly, it is possible to fit the nonmonotonic relaxation curves to a modified version Eq. (3), namely, $V_{3}(t)=A+B\left[\left(F_{3}-F_{2}\right) \log (t)+\left(F_{2}-F_{1}\right)\right.$ $\left.\log \left(t+C t_{w}\right)\right]$, using $A$ as a fitting parameter and with $B=A \times 0.025$ and $C=2.55$ for all curves. Such fits are shown for different values of $t_{w}$ and $\Delta$ in Fig. 5(b).

As shown earlier, the single-step relaxation of the elastic foams can be described by a superposition of two logarithmic decays, offset by a time $t_{0}$ that depends on the compression. In analogy to Eq. (3), one can try to describe the nonmonotonic behavior in the elastic foams using a superposition of four logarithmic processes. Indeed, it can be shown that this introduces only small corrections to the linear scaling between $t_{p}$ and $t_{w}$, in agreement with the data collapse in Fig. 5(a). However, due to the nonlinearity introduced by the compression-dependent $t_{0}$, it is not possible to use the single-step relaxations to obtain a good fit to the nonmonotonic relaxation curve.

The nonmonotonic relaxations reported here are reminiscent of the aging behavior first described in the pioneering work of Kovacs [17]. Kovacs examined the slow volume changes of polymer melts following a temperature change, demonstrating memory retention in a glassy system. Analogous phenomena were observed in the time-dependant viscosity of metallic glasses [39] and the density of agitated granular systems [19] as well as in numerical studies [40,41]. Despite recent progress [42-46], this phenomenon is still not well understood. Our observations and their agreement with a phenomenological framework known to describe relaxation and aging in glassy systems are clear evidence that athermal mechanical systems can exhibit glassy dynamics and that the 
nonmonotonic behavior described here may be generic to many disordered systems. Techniques such as direct visualization [47-49] and monitoring of acoustic emission $[50,51]$ combined with a physical understanding of the systems used in this study [52-54] may shed new light on the structural origin of the slow relaxation, nonmonotonic aging, and memory effects observed in these systems.

We thank F. Spaepen, Y. Oreg, Y. Imry, J. P. Bouchaud, and T. A. Witten for illuminating discussions. This work was supported by the National Science Foundation through the Harvard Materials Research Science and Engineering Center (DMR-1420570). A. A. acknowledges support from the Milton Fund. A. A. and S. M. R. acknowledge support from the Alfred P. Sloan research foundation.

[1] T. Grenet, J. Delahaye, M. Sabra, and F. Gay, Anomalous electric-field effect and glassy behaviour in granular aluminium thin films: electron glass?, Eur. Phys. J. B 56, 183 (2007).

[2] A. Vaknin, Z. Ovadyahu, and M. Pollak, Aging Effects in an Anderson Insulator, Phys. Rev. Lett. 84, 3402 (2000).

[3] M. Pollak, M. Ortuño, and A. Frydman, The Electron Glass (Cambridge University Press, Cambridge, England, 2013).

[4] A. Amir, S. Borini, Y. Oreg, and Y. Imry, Huge (but Finite) Time Scales in Slow Relaxations: Beyond Simple Aging, Phys. Rev. Lett. 107, 186407 (2011).

[5] A. Amir, Y. Oreg, and Y. Imry, Electron glass dynamics, Annu. Rev. Condens. Matter Phys. 2, 235 (2011).

[6] Y. Kim, C. Hempstead, and A. Strnad, Critical Persistent Currents in Hard Superconductors, Phys. Rev. Lett. 9, 306 (1962).

[7] P. Anderson, Theory of Flux Creep in Hard Superconductors, Phys. Rev. Lett. 9, 309 (1962).

[8] L. F. Cugliandolo and J. Kurchan, Analytical Solution of the Off-Equilibrium Dynamics of a Long-Range Spin-Glass Model, Phys. Rev. Lett. 71, 173 (1993).

[9] J.-P. Bouchaud, L. Cugliandolo, J. Kurchan, and M. Mezard, Out of equilibrium dynamics in spin-glasses and other glassy systems, in Spin Glasses and Random Fields (World Scientific Publishing, Singapore, 1998), pp. 161-223.

[10] H. E. Castillo, C. Chamon, L. F. Cugliandolo, and M. P. Kennett, Heterogeneous Aging in Spin Glasses, Phys. Rev. Lett. 88, 237201 (2002).

[11] V. Dupuis, F. Bert, J.-P. Bouchaud, J. Hammann, F. Ladieu, D. Parker, and E. Vincent, Aging, rejuvenation and memory phenomena in spin glasses, Pramana 64, 1109 (2005).

[12] E. R. Weeks, J. C. Crocker, A. C. Levitt, A. Schofield, and D. A. Weitz, Three-dimensional direct imaging of structural relaxation near the colloidal glass transition, Science $\mathbf{2 8 7}$, 627 (2000).

[13] L. Cipelletti, E. R. Weeks et al., in Dynamical Heterogeneities in Glasses, Colloids, and Granular Media (Oxford University Press, Oxford, 2011).

[14] S. M. Rubinstein, G. Cohen, and J. Fineberg, Contact Area Measurements Reveal Loading-History Dependence of Static Friction, Phys. Rev. Lett. 96, 256103 (2006).
[15] S. Rubinstein, G. Cohen, and J. Fineberg, Visualizing stickslip: experimental observations of processes governing the nucleation of frictional sliding, J. Phys. D 42, 214016 (2009).

[16] O. Ben-David, S. M. Rubinstein, and J. Fineberg, Slip-stick and the evolution of frictional strength, Nature (London) 463, 76 (2010).

[17] A. J. Kovacs, Glass transition in amorphous polymers: a phenomenological study, Adv. Polym. Sci. 3, 394 (1963).

[18] L. C. E. Struik, Physical aging in plastics and other glassy materials, Polym. Eng. Sci. 17, 165 (1977).

[19] J. B. Knight, C. G. Fandrich, C. N. Lau, H. M. Jaeger, and S. R. Nagel, Density relaxation in a vibrated granular material, Phys. Rev. E 51, 3957 (1995).

[20] R. Albuquerque and M. Gomes, Stress relaxation in crumpled surfaces, Physica (Amsterdam) 310A, 377 (2002).

[21] K. Matan, R. B. Williams, T. A. Witten, and S. R. Nagel, Crumpling a Thin Sheet, Phys. Rev. Lett. 88, 076101 (2002).

[22] R. G. Palmer, D. L. Stein, E. Abrahams, and P. W. Anderson, Models of Hierarchically Constrained Dynamics for Glassy Relaxation, Phys. Rev. Lett. 53, 958 (1984).

[23] P. Sibani and K. H. Hoffmann, Hierarchical models for aging and relaxation of spin glasses, Phys. Rev. Lett. 63, 2853 (1989).

[24] J.-P. Bouchaud, Weak ergodicity breaking and aging in disordered systems, J. Phys. I (France) 2, 1705 (1992).

[25] J.-P. Bouchaud, in Soft and Fragile Matter: Nonequilibrium Dynamics, Metastability and Flow. edited by M. E. Cates and M. R. Evans (IOP Publishing, Bristol and Philadelphia, 2000), pp. 285-304.

[26] I. Kolvin and E. Bouchbinder, Simple nonlinear equation for structural relaxation in glasses, Phys. Rev. E 86, 010501 (2012).

[27] A. Amir, Y. Oreg, and Y. Imry, On relaxations and aging of various glasses, Proc. Natl. Acad. Sci. U.S.A. 109, 1850 (2012).

[28] A. S. Balankin, O. Susarrey Huerta, F. Hernandez Mendez, and J. Patino Ortiz, Slow dynamics of stress and strain relaxation in randomly crumpled elasto-plastic sheets, Phys. Rev. E 84, 021118 (2011).

[29] O. Gottesman and S. M. Rubinstein (to be published).

[30] G. A. Vliegenthart and G. Gompper, Forced crumpling of self-avoiding elastic sheets, Nat. Mater. 5, 216 (2006).

[31] W. Bai, Y.C. Lin, T. K. Hou, and T. M. Hong, Scaling relation for a compact crumpled thin sheet, Phys. Rev. E 82, 066112 (2010).

[32] S. Deboeuf, E. Katzav, A. Boudaoud, D. Bonn, and M. Adda-Bedia, Comparative Study of Crumpling and Folding of Thin Sheets, Phys. Rev. Lett. 110, 104301 (2013).

[33] T. Baumberger and C. Caroli, Solid friction from stick-slip down to pinning and aging, Adv. Phys. 55, 279 (2006).

[34] A. Van Der Ziel, On the noise spectra of semi-conductor noise and of flicker effect, Physica (Amsterdam) 16, 359 (1950).

[35] A. Amir, Y. Oreg, and Y. Imry, 1/f noise and slow relaxations in glasses, Ann. Phys. (Berlin) 18, 836 (2009).

[36] J. J. Waterfall, F. P. Casey, R. N. Gutenkunst, K. S. Brown, C. R. Myers, P. W. Brouwer, V. Elser, and J. P. Sethna, Sloppy-Model Universality Class and the Vandermonde Matrix, Phys. Rev. Lett. 97, 150601 (2006). 
[37] X. Cao, A. Rosso, J.-P. Bouchaud, and P. L. Doussal, Genuine localisation transition in a long-range hopping model, arXiv:1607.04173.

[38] C. W. Beenakker, Random-matrix theory of quantum transport, Rev. Mod. Phys. 69, 731 (1997).

[39] C. Volkert and F. Spaepen, Crossover relaxation of the viscosity of $\mathrm{Pd}_{40} \mathrm{Ni}_{40} \mathrm{P}_{19} \mathrm{Si}_{1}$ near the glass transition, Acta Metall. 37, 1355 (1989).

[40] S. Mossa and F. Sciortino, Crossover (or Kovacs) Effect in an Aging Molecular Liquid, Phys. Rev. Lett. 92, 045504 (2004).

[41] L. Cugliandolo, G. Lozano, and H. Lozza, Memory effects in classical and quantum mean-field disordered models, Eur. Phys. J. B 41, 87 (2004).

[42] E. Bertin, J. Bouchaud, J. Drouffe, and C. Godreche, The Kovacs effect in model glasses, J. Phys. A 36, 10701 (2003).

[43] A. Prados and J. Brey, The Kovacs effect: a master equation analysis, J. Stat. Mech.: Theory Exp. (2010) P02009.

[44] G. Diezemann and A. Heuer, Memory effects in the relaxation of the Gaussian trap model, Phys. Rev. E 83, 031505 (2011).

[45] E. Bouchbinder and J. Langer, Nonequilibrium thermodynamics of the Kovacs effect, Soft Matter 6, 3065 (2010).
[46] A. Prados and E. Trizac, Kovacs-Like Memory Effect in Driven Granular Gases, Phys. Rev. Lett. 112, 198001 (2014).

[47] H. Aharoni and E. Sharon, Direct observation of the temporal and spatial dynamics during crumpling, Nat. Mater. 9, 993 (2010).

[48] A. D. Cambou and N. Menon, Three-dimensional structure of a sheet crumpled into a ball, Proc. Natl. Acad. Sci. U.S.A. 108, 14741 (2011).

[49] B. Thiria and M. Adda-Bedia, Relaxation Mechanisms in the Unfolding of Thin Sheets, Phys. Rev. Lett. 107, 025506 (2011).

[50] P. A. Houle and J.P. Sethna, Acoustic emission from crumpling paper, Phys. Rev. E 54, 278 (1996).

[51] E. M. Kramer and A. E. Lobkovsky, Universal power law in the noise from a crumpled elastic sheet, Phys. Rev. E 53, 1465 (1996).

[52] T. Witten, Stress focusing in elastic sheets, Rev. Mod. Phys. 79, 643 (2007).

[53] D. Nelson and L. Radzihovsky, Polymerized membranes with quenched random internal disorder, Europhys. Lett. 16, 79 (1991).

[54] N. Oppenheimer and T. A. Witten, Shapeable sheet without plastic deformation, Phys. Rev. E 92, 052401 (2015). 